\title{
Embolisation of Bleeding Renal Angiomyolipoma in Pregnancy
}

\author{
Chin Hong Lim, David Mulvin \\ Department of Urology, St. Vincent's University Hospital, Dublin, Ireland \\ E-mail: chinhong242002@yahoo.com \\ Received May 10, 2011; revised June 18, 2011; accepted June 25, 2011
}

\begin{abstract}
Bleeding from renal angiomyolipoma in pregnancy can be catastrophic to both mother and fetus. Selective arterial embolisation is considered more superior than conventional surgery in these patients. Our case report exemplifies how a bleeding angiomyolipoma was halted with embolisation in our pregnant patient.
\end{abstract}

Keywords: Selective Arterial Embolisation, Renal Angiomyolipoma, Pregnancy, Bleeding, Choristoma

\section{Introduction}

Renal angiomyolipoma (AML) was once considered a rare tumour with the diagnosis traditionally established by histology after removal of the renal lesion, it is now recognised as a relatively common lesion with a broad range of clinical sequelae that is amenable to different management options. Recent developments in interventional radiology have provided new tools for diagnosing and treating this interesting condition.

\section{Case Report}

A 32-year-old female gravida 3, para 2 presented with left flank pain radiating to her left groin at 24 weeks gestation. Her haemoglobin dropped from $10.7 \mathrm{~g} / \mathrm{dL}$ on admission to $6.9 \mathrm{~g} / \mathrm{dL}$ over 24 hours. On examinations, the fundus of her uterus palpable consistent with her gestation and there was fullness and tenderness over her left renal angle. Investigation revealed haemoglobin of $10.3 \mathrm{~g} / \mathrm{dL}$, WCC $11.3 \times 10^{9}$, platelets $186 \times 10^{9}$ and normal renal function (urea $2.1 \mu \mathrm{mol} / \mathrm{l}$, creatinine $49 \mu \mathrm{mol} / \mathrm{l}$ ). Her ultrasound showed $7 \times 8 \mathrm{~cm}$ echogenic lesion in her left kidney with hyperechoic component suggestive of angiomyolipoma (Figure 1). She was haemodynamically stable after transfusion of 2 units of red cells. On day 3 post-admission, she developed pain and her Hb dropped from $10.7 \mathrm{~g} / \mathrm{dL}$ to $8.6 \mathrm{~g} / \mathrm{dL}$. She underwent urgent MRI. But because of her claustrophobia, only T1 axial sequences and localisers could be obtained. This confirms the presence of a large (approximately $12 \mathrm{~cm}$ ) left-sided abdominal mass with a high signal within it consistent with fat or blood (Figure 2). The overall MR appearances are entirely consistent with the presumptive clinical diagnosis of a bleed into a left renal tumour, most likely angiomyolipoma. It was elected to proceed to embolisation of bleeding angiomyolipoma.

An initial aortogram was performed via the left femoral artery to assess the number and location of left renal artery. Selective renal arteriography was then used to delineate the vascular anatomy of the target tumour. The feeding vessel was then selectively catheterised with microcatheter (Figure 3(a)). Embolisation was performed with combination of microspheres (300 - 500 microns) (Terumo Europe N.V.) and coil (3 mm) (manufactured by William Cook Europe) positioned in actively bleeding vessel. Post-embolisation film showed excellent result. (Figure 3(b)).

Following the procedure, the patient complained of left sided pleuritic chest pain and palpitations. Spiral CT revealed no evidence of pulmonary embolism and her symptoms settled with simple oral analgesia. Her repeat Haemoglobin for the next 5 days was stable and she was discharged on day 6 post-embolisation.

The proceeding pregnancy was completely uneventful and at 40 weeks she gave birth by elective caesarean section to a normal female infant weighting $3000 \mathrm{~g}$. There were no further complications during the pregnancy or in the post-partum period.

She was reviewed on outpatient clinic 8 weeks post partum complaint of ongoing left flank pain. CT abdomen showed a $10 \times 4 \times 4 \mathrm{~cm}$ collection in left renal bed. The lesion was not suitable for re-embolisation and decision was make to remove the left kidney. She had elective 


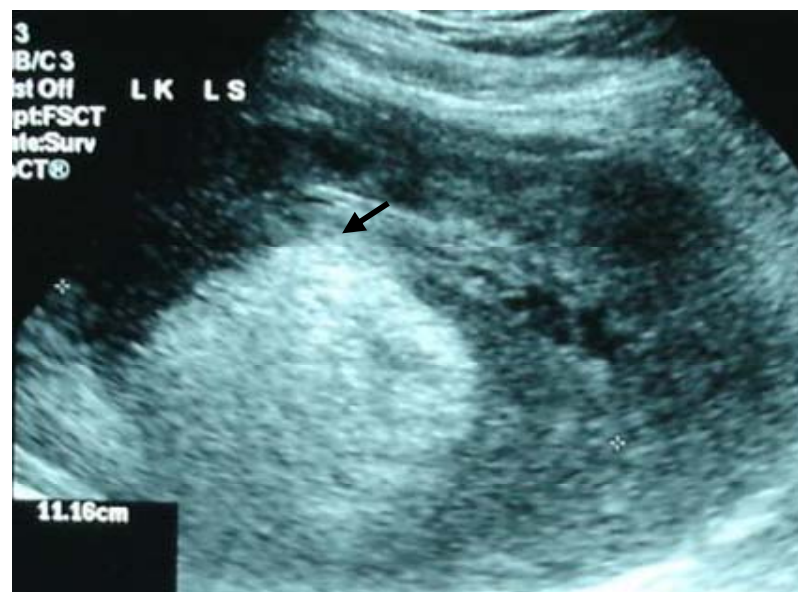

Figure 1. Ultrasound showed echogenic lesion with hyperechoic component suggestive of angiomyolipoma.

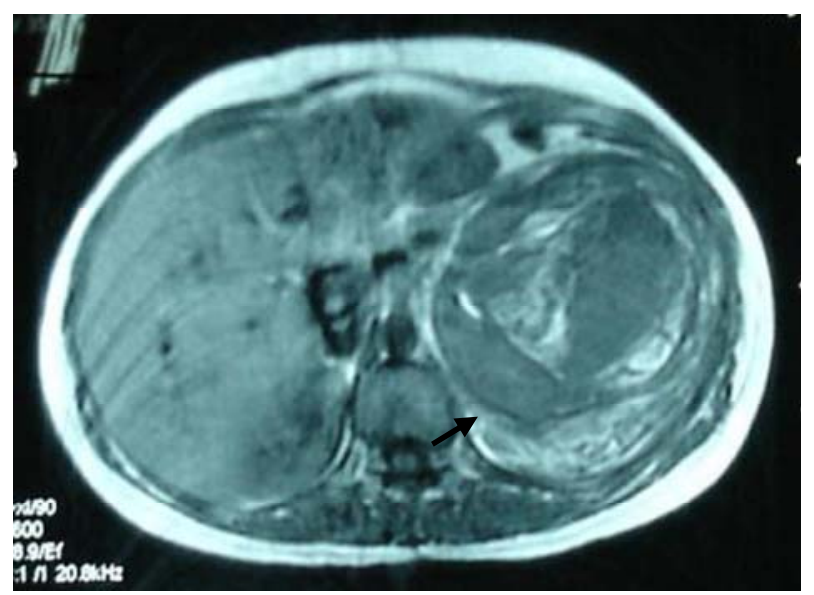

Figure 2. T1 axial sequences showing a large (approximately $12 \mathrm{~cm}$ ) left-sided abdominal mass with a high signal within it consistent with fat or blood.

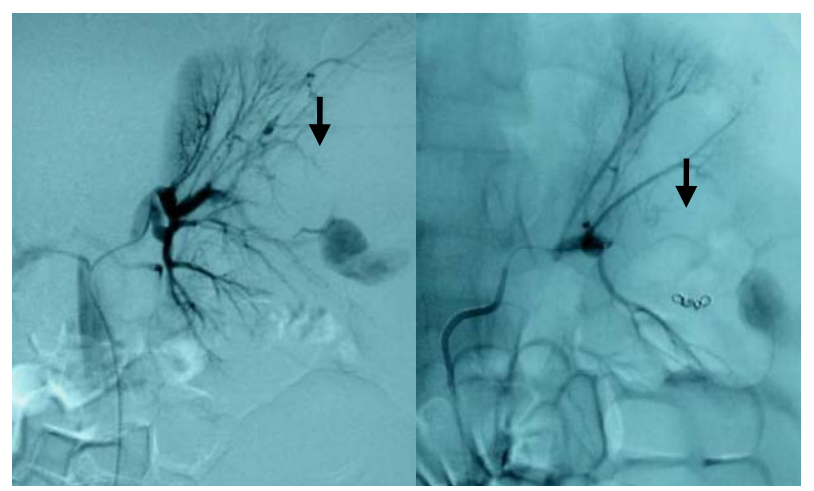

(a)

(b)

Figure 3. Selective injection into the single renal artery at the point of its division into two equal-sized branches. Note the branch feeding the vascular malformation. Post-embolisation showing the stasis of contrast beyond the coils.

open left nephrectomy and discharged 10 days later.

\section{Discussion}

The term angiomyolipoma (AML) is attributed to Morgan et al. in 1951 [1], the renal lesion that histological corresponds to a renal AML was first described by Grawitz in 1900 [2]. Angiomyolipoma is composed of adipose tissue, smooth muscle and blood vessels in various proportions. Angiomyolipoma is composed of different proportions of adipose tissue, smooth muscle, blood vessel as a result of abnormal proliferation. Occasionally it has also been referred to as choristoma due to the presence of tissue type which did not originated from the kidney. Irrespective of labels angiomyolipoma is increasingly understood to be a neoplastic but benign process based on its clinical sequelae and molecular characteristics.

AML appears in two distinctive settings, as part of the tuberous sclerosis complex (TSC) and sporadically in individuals who otherwise have no clinical features of that entity. In the former group, these tumours are often bilateral, have multicentric foci and tend to bleed more often than sporadic variant $[3,4]$. Steiner et al. demonstrated that this group have worse outcome with faster growth rates (67\% versus 21\%) and earlier need for operating intervention (50\% versus 28\%) [5].

The sporadic type AML is in comparison a slower growing neoplasm with better outcome. However, even in this form, female or pregnant patients tended to have a more complicated AML. In fact, L'Hostis et al. and Henske at al discovered the presence of progesterone and oestrogen receptors in these tumours [6,7].

Although most AMLs are small and asymptomatic, some authors suggested that up to $80 \%$ of patients when tumour size reaches $4 \mathrm{~cm}$ or greater, half of them present with abdominal or flank pain and $10 \%$ - 20\% with haematuria or palpable mass [8]. The tendency to rupture has been attributed to the weak, elastin poor vascular structures in the tumour. This presentation appears to occur during the physiological changes of pregnancy.

Treatment options for AML include total or partial nephrectomy, cryoablation, radiofrequency ablation and transcatheter selective arterial embolisation. Since the first report of angiographic AML embolisation, this technique has become common for managing this entity. The principal advantages of selective embolisation for AML include the preservation of functional renal parenchyma, ability to embolise bleeding vessels selectively and circumvention of need for a surgical excision or general anaesthesia. The most common indication for embolisation has been acute haemorrhage due to spontaneous tumour rupture. Other indications include symptomatic tumour in patients who are poor operative candidates or have limited renal reserve. With our patient, 
because of her pregnancy, operative intervention would be best avoided. Hence, we chose embolisation of the tumour to control the bleeding while waiting for the gestation to finish.

In the literature, complications after embolisation were reported in $10 \%$ of cases, the most common being abscess formation (5\%) and pleural effusion (3\%). A single death from respiratory failure was reported in a patient with severe pre-existing lung disease. $85 \%$ of patients experienced a post-embolisation syndrome of flank pain, fever, leukocytosis and nausea attributable to inflammatory mediators. Treatment is supportive with antipyretics, antiemetics and analgesics and most patients recover in 2 to 5 days [9].

Spontaneous renal haemorrhage during pregnancy is uncommon but consequences may be catastrophic. The first case of AML associated with bleeding in pregnancy was reported in 1964. A total of 16 cases have since been published with only two associated with tuberous sclerosis. 12 of the 16 cases required surgical intervention during the pregnancy while the other 4 had embolisation. One woman had embolisation prior to becoming pregnant but had two episodes of rebleeding during pregnancy. She was managed conservatively and completed the pregnancy without any complications. The other women completed a normal pregnancy and had embolisation 7 days post-partum. Two patients had embolisation during pregnancy at 28 and 12 weeks respectively [10].

In conclusion, selective arterial embolisation is an attractive alternative to surgical intervention for spontaneous haemorrhage from AML during pregnancy. As with patient, ongoing symptoms and high possibility of rebleeding (large tumour), nephrectomy may be required post partum.

\section{References}

[1] G. S. Morgan, J. V. Straumfjord and E. J. Hall, “Angio- myolipoma of the Kidney,” Journal of Urology, Vol. 65, 1951, pp. 525-527.

[2] P. Grawitz, "Demonstration Eines Grossen Angio-MyoLipomas der Niere,” Dtsch Med Wochenschr, Vol. 26, 1900, p. 290.

[3] T. J. Stillwell, M. R. Gomez and P. P. Kelalis, "Renal Lesions in Tuberous Sclerosis,” Journal of Urology, Vol. 138, 1987, p. 477.

[4] J. Bernstein, T. O. Robbins and J. M. Kissane, "The Renal Lesion in Tuberous Sclerosis,” Seminars in Diagnostic Pathology, Vol. 3, 1985, p. 97.

[5] M. S. Steiner, S. M. Goldman, F. E. K. ishman and F. F. Marshall, "The Natural History of Renal Angiomyolipoma,” Journal of Urology, Vol. 150, 1993, p. 1782.

[6] H. L'Hostis, C. Deminiere and J. M. Ferreire, "Spontaneous Haemorrhage during Pregnancy Secondary to Renal Angiomyolipoma,” International Journal of Urology, Vol. 56, 1999, pp. 188-191.

[7] E. P. Henske, M. P. Short, R. Greenberg, H. P. Neumann and D. J. Kwiatkowski, "Frequent Progesterone Receptor Immunoreactivity in Tuberous Sclerosis Associated Renal Angiomyolipomas,” Modern Pathology, Vol. 11, 1998, p. 665.

[8] J. E. Oesterling, E. K. Fishman and S. M. Goldman, "The Management of Renal Angiomyolipoma," Journal of Urology, Vol. 135, No. 6, 1986, pp. 1121-1124.

[9] J. A. Hamlin, D. C. Smith, F. C. Taylor, J. M. McKinney, H. C. Ruckle and H. R. Hadley, "Renal Angiomyolipomas: Long-Term Follow-up of Embolisation for Acute Haemorrhage,” Journal of Radiology, Vol. 48, 1997, p. 191.

[10] J. Shah, J. Jones and M. A. W. Miller, "Selective Embolisation of Bleeding Renal Angiomyolipoma in Pregnancy," Journal of the Royal Society of Medicine, Vol. 92, 1999, pp. 414-415. 